\title{
Rate Capability of Lithium Intercalation into Nano-porous
}

\section{Graphitized Carbons}

Hirotoshi Yamada, ${ }^{1}$ Yuko Watanabe, ${ }^{2}$ Isamu Moriguchi, ${ }^{1}$ Tetsuichi Kudo ${ }^{3}$

${ }^{1}$ Faculty of Engineering, and ${ }^{2}$ Graduate School of Science and Technology, Nagasaki University, 1-14, Bunkyo-machi, Nagasaki 852-8521, Japan

${ }^{3}$ National Institute of Advanced Industrial Science and Technology, Tsukuba Central 2, 11-1, Umezono, Tsukuba, 305-8568, Japan

email: h-yama@nagasaki-u.ac.jp

\begin{abstract}
Nanoporous graphitized carbons were successfully prepared by using mono-dispersed $\mathrm{SiO}_{2}$ colloidal crystal as a template and mesophase pitch as a carbon source with final heat treatment temperatures (HTT) of $1000-2500{ }^{\circ} \mathrm{C}$. Rate capability of lithium intercalation/deintercalation of the nano-porous graphitized carbons was investigated. $35-60 \%$ of capacities were retained when the current density was increased from $37.2 \mathrm{~mA} \mathrm{~g}^{-1}$ to $372 \mathrm{~mA} \mathrm{~g}^{-1}$. Electrochemical impedance spectra indicated that formation of SEI layers caused increased polarization.
\end{abstract}

Keywords: Lithium ion battery, Porous carbon, Graphite 


\section{Introduction}

Development of energy storage devices with both large capacity and high power are accelerated to satisfy the growing demands from their application not only to multi-function portable electronic devices but also to electric vehicles. Lithium ion batteries (LIBs) are one of the most promising candidates of such power sources. Although energy density of LIBs is the largest among present rechargeable devices, their power density is not enough. Their relatively low power density is explained by the following polarizations on the electrode reactions: (1) charge transfer reaction, (2) lithium ion diffusion in solid active materials, (3) electron conduction and (4) electrolyte transport. We have so far proposed and demonstrated that rate capability of lithium ion batteries (LIB) is highly improved by using nano-porous materials (e.g., $\mathrm{TiO}_{2},[1,2] \mathrm{V}_{2} \mathrm{O}_{5}$, [3] $\mathrm{LiFePO}_{4}$ ). These materials may be applicable to cathodes of LIB. As a next step of the study, we focused on anode materials. It is well known that graphitic carbons are used as anodes on the basis of the following reversible reaction: $x \mathrm{Li}^{+}+$ $x \mathrm{e}^{-}+6 \mathrm{C} \leftrightarrow \operatorname{Li}_{x} \mathrm{C}_{6}(0 \leq x \leq 1)$. For $x=1$, graphitic carbons exhibit a maximum capacity of $372 \mathrm{~mA} \mathrm{~h} \mathrm{~g}^{-1}$. Lithium intercalation into graphite is affected not only by factors listed above, but also by solid electrolyte interface (SEI) layers that are formed on the graphite surface by decomposition of electrolytes. Nano-porous graphitized carbons with large surface area are expected to exhibit small polarization on lithium intercalation. In recent studies, we have fabricated nano-porous carbons and evaluated them as electrodes for electric double layer capacitors. The nano-porous carbons prepared from phenolic resin exhibited superb performance due to their large specific surface area and controlled pores.[4,5] We have also succeeded in preparation of nano-porous graphitized carbons and evaluated their Liintercalation properties, which were characterized by three features: (1) large capacity, (2) large capacity hysteresis, and (3) decreasing capacity with increasing HTT. The first and 
second features are explained by micropores and the third one is supposed to result from graphene layer orientation on pore surfaces. In this study, we focused on the rate capability of lithium intercalation into the nano-porous graphitized carbons.

\section{Experimental}

Nano-porous graphitize carbons were synthesized by a colloidal-crystal templating method.[3-5] First, $\mathrm{SiO}_{2}$ colloidal crystal with an average diameter of $80 \mathrm{~nm}$ was yielded by centrifugation. Then, coal-derived mesophase pitch was impregnated into the interstices among spherical $\mathrm{SiO}_{2}$ particles. After carbonization of the mesophase pitch at $1000{ }^{\circ} \mathrm{C}$ in $\mathrm{Ar}$ and subsequent $\mathrm{SiO}_{2}$ removal with $\mathrm{HF}$ aqueous solution, nano-porous carbons were synthesized. Finally, by heating the nano-porous carbons at $1500-2500{ }^{\circ} \mathrm{C}$ in Ar, nano-porous graphitized carbons were obtained. Hereafter, specimen will be denoted as C[80]-HTT where HTT is the final heat treatment temperature $\left(1000,1500,2000\right.$, and $\left.2500{ }^{\circ} \mathrm{C}\right)$. For comparison, non-porous graphitized carbons were prepared from the same carbon sources without templates, which are abbreviated as C[non]-HTT.

Structure of carbon was examined by X-ray diffraction (XRD) on a RINT-2200 (Rigaku, with irradiation of Ni-filtered $\mathrm{CuK} \alpha$ ) and Raman spectroscopy on a RMP-210 (JASCO, with a 532-nm laser). Porous structure was observed by transmission electron microscopy (TEM, JEOL JEM-2010) and pore parameters were obtained by nitrogen adsorption/desorption isotherms at $77 \mathrm{~K}$ recorded on a BELSORP-mini (BEL Japan, Inc.). Lithium storage properties of nanoporous carbons were galvanostatically investigated on an electrochemical analyzer HJ-SM8 (Hokuto Denko Corp.) in the potential range from 3.0 to $0.01 \mathrm{~V}$ vs. $\mathrm{Li} / \mathrm{Li}^{+}$at $25{ }^{\circ} \mathrm{C}$. As working electrodes, porous carbons were mixed with PTFE at a mass ratio of 95:5 and were pressed onto Ni nets. Both reference and counter electrodes 
employed metallic lithium pressed on Ni nets. A $1 \mathrm{M} \mathrm{LiPF}_{6}$ solution in ethylene carbonate (EC) and dimethyl carbonate (DMC) (1:1 by volume) was used as an electrolyte. The working electrodes were immersed in the electrolyte in vacuo beforehand. In this paper, the terms "charge" and "discharge" are defined as the cathodic (i.e., Li intercalation to graphene layers) and anodic (Li de-intercalation) processes, respectively. The cell was assembled in an Arfilled glove box in which $\mathrm{H}_{2} \mathrm{O}$ concentration was kept below 1 ppm.

\section{Results and Discussion}

\subsection{STRUCTURAL CHARACTERIZATION}

Thermal gravimetry confirmed that the residual $\mathrm{SiO}_{2}$ was less than 2-3\% for C[80]-1000. XRD patterns (see Fig. 1(a) in Ref. 6) demonstrated that with increasing HTT, diffraction peaks become sharper, which means the progress of graphitization. But (002) diffraction peaks ( 26 degree) exhibited broad and asymmetric bottoms, indicating the distribution of the degree of graphitization to some extent. For C[non]-HTTs, (002) peaks were symmetric, and the asymmetric peaks are originated from the porous structure. Raman spectra (not shown) exhibited both D-band $\left(\sim 1350 \mathrm{~cm}^{-1}\right)$ and G-band $\left(\sim 1580 \mathrm{~cm}^{-1}\right)$ for all C[80]-HTTs. The ratio of the peak intensity $I_{\mathrm{G}} / I_{\mathrm{D}}$ increased with increasing HTT but still 0.9 for $\mathrm{C}[80]-2500$. This result also indicates that crystallization is on the way. In TEM images (Fig. 2), for C[80]-1000, three-dimensionally ordered spherical pores with a diameter of c.a. $80 \mathrm{~nm}$ was observed. With increasing HTT, pore diameter reduced resulting from the sintering on the graphitization. For $C[80]-2000$ and $C[80]-2500$, graphene layers were observed on the pore walls (Fig. 2(e,f)). $\mathrm{N}_{2}$ adsorption/desorption isotherms exhibited steep increasing of $\mathrm{N}_{2}$ uptake around the relative pressure of $0.9-1.0$, which means the $\mathrm{N}_{2}$ condensation in relatively large pores. From the adsorption branches, specific surface areas were obtained. In this study, total surface area 
$\left(S_{\text {total }}\right)$ and meso/macropore surface area $\left(S_{\text {meso }}\right)$ were obtained from $\alpha_{\mathrm{SPE}}$-plot and $t$-plot, respectively. Micorpore surface area $\left(S_{\text {micro }}\right)$ was given by subtracting $S_{\text {meso }}$ from $S_{\text {total }}$. The specific surface areas are summarized in table 1. It should be noted that nano-porous graphitized carbons exhibited not only large $S_{\text {meso }}$ but also considerable $S_{\text {micro }}$. In the case of phenolic-resin-derived carbons, micropores are originated from interstices of graphene layers formed on carbonization, and thus, $S_{\text {micro }}$ depends on carbon sources and carbonization condition. The template dependence of $S_{\text {micro }}$ in this study is explained by orientation mismatch of the mesophase pitch in the interstices of $\mathrm{SiO}_{2}$ particles on carbonization. Jian et al. have reported that mesophase pitch exhibit orientation preference on the surfaces, and on $\mathrm{SiO}_{2}$, edge of graphene layers anchors to the surface.[6] This orientation preference would result in orientation mismatch of mesophase pitch, which gives rise to interstices and distortion between mesophase pitch, and finally micropores and poorly crystallized domains are formed.

\subsection{LITHIUM INTERCALATION}

Galvanostatic potential curves of nano-porous graphitized carbons were recorded with different current densities (37.2, 186 and $372 \mathrm{~mA} \mathrm{~g}^{-1}$ ). The nano-porous graphitized carbons were subjected to lithium intercalation and de-intercalation for three cycles at each current density. The galvanostatic curves of the 3rd cycle at each current density are shown in Fig. 2 . Discharge capacities at $372 \mathrm{~mA} \mathrm{~g}^{-1}$ was as much as $49 \%, 58 \%$ and $36 \%$ of those at $372 \mathrm{~mA}$ $\mathrm{g}^{-1}$ for $\mathrm{C}[80]-1000, \mathrm{C}[80]-2000$, and $\mathrm{C}[80]-2500$, respectively. The rate capability of the nano-porous graphitized carbons was not so good as expected, which was comparable or slightly higher than that of conventional MCMB, but lower than graphitized carbon nanobeads. [7] The relatively poor rate capability of the nano-porous graphitized carbons is 
partially given rise to the fact that the lithium insertion potential is very close to the lower cutoff potential $\left(0.01 \mathrm{~V}\right.$ vs. $\left.\mathrm{Li} / \mathrm{Li}^{+}\right)$. When large current densities were applied on lithium intercalation, potential curves shift to bottom due to polarization on electrochemical processes, and potential reaches the cut-off potential although accumulated lithium ions are few. In order to analyze the causes of the polarization, electrochemical impedance spectra (Fig. 3) were recorded at the 1 st discharge process and after the galvanostatic charge tests, i.e., at the 10th cycles. In the impedance spectra of the initial electrode (3.0 V, solid circle), a semicircle and a vertical line were observed, which is the typical plot of electric double layer capacitors. After the 1st lithium intercalation $(0.01 \mathrm{~V}$, solid triangle $)$, the plot exhibited a semi-circle and a sloped line, indicating the finite length diffusion process. From the width of the sloped line, the Warburg impedance was almost comparable with the charge transfer resistance (the diameter of the semicircle), which means that Li-ion diffusion in carbon is relatively fast due to the short diffusion length. For the electrode that experienced 9-cycle intercalation/deintercalation process, a large semicircle with a long sloped line was observed at $3.0 \mathrm{~V}$. It is obvious that the diameter of the semicircle increased with increasing the cycle number, e.g., 5 $\Omega$ to $12 \Omega$ for the Nyquist plots at $0.01 \mathrm{~V}$. This is explained by the SEI layers formed on carbon surfaces with a time constant overlapped with that of the charge transfer process. It should be also mentioned that the intercept of the semicircles at high frequency shifted toward right. This is explained by the increasing electrolyte resistance, resulting from increase in thickness and/or density of the SEI layers. For EC containing electrolyte, it is reported that the SEI layers are porous and thick $(\sim 40 \mathrm{~nm})$, and increases in thickness with repeating lithium intercalation/de-intercalation cycles.[8] After several cycles, SEI layers are supposed to be dominant in pores and to hinder smooth electrolyte transport in pores. 


\section{Conclusions}

In this paper, we investigated the rate capability of the nano-porous graphitized carbons. The discharge capacity of the nano-porous graphitized carbons decreased with increasing current densities. The capacities at $372 \mathrm{~mA} \mathrm{~g}^{-1}$ were $35-60 \%$ of those at $37.2 \mathrm{~mA} \mathrm{~g}^{-1}$. Electrochemical impedance spectra indicated that lithium ion diffusion in carbon is relatively fast due to the short diffusion length for the nano-porous graphitized carbons. The relatively poor rate capability was caused by the formation of SEI layers on pore surfaces. It was found that the SEI layers increases the polarization in two ways: resistance of SEI layers and hindering electrolyte transport. It is important to control SEI layers with adequate additives [9] or to design the porous structure that is large enough in size to facilitate electrolyte transport through SEI layers.

\section{References}

[1] H. Yamada, T. Yamato, I. Moriguchi, T. Kudo, Solid State Ionics, 175 (2004) 195.

[2] I. Moriguchi, R. Hidaka, H. Yamada, T. Kudo, H. Murakami, N. Nakashima, Adv. Mater, $18(2006) 69$.

[3] H. Yamada, K. Tagawa, M. Komatsu, I. Moriguchi, T. Kudo, J. Phys. Chem. C, 111 (2007) 8397.

[4] H. Yamada, H. Nakamura, F. Nakahara, I. Moriguchi, T. Kudo, J. Phys. Chem. C, 111 (2007) 227.

[5] H. Yamada, I. Moriguchi, T. Kudo, J. Power Sources, 175 (2008) 651.

[6] K. Jian, H.-S. Shim, D. Tuhus-Dubrow, S. Bernstein, C. Woodward, M. Pfeffer, D. Steingart, T. Gournay, S. Sachsmann, G. P. Crawford, R. H. Hurt, Carbon, 41 (2003) 2073. 
[7] H. Wang, T. Abe, S. Maruyama, Y, Iriyama, Z. Ogumi, K. Yoshikawa, Adv. Mater., 17 (2005) 2857.

[8] S.-K. Jeong, M. Inaba, T. Abe, Z. Ogumi, J. Electrochem. Soc., 148 (2001) A989.

[9] S.-K. Jeong, M. Inaba, R. Mogi, Y. Iriyama, T. Abe, Z. Ogumi, Langmuir, 17 (2001) 8281. 
Table 1. Specific surface areas of nano-porous graphitized carbons. Numbers in parentheses are the ratio of meso- (macro-) and micropore surface area to total surface area.

$$
\text { Specific surface area }\left(\mathrm{m}^{2} \mathrm{~g}^{-1}\right)
$$

\begin{tabular}{llll}
\hline Samples & Total & Meso\&Macro & Micro \\
\hline $\mathrm{C}[$ non $]$ & $<7$ & $<2$ & $<5$ \\
$\mathrm{C}[80]-1000$ & 586 & $511(87 \%)$ & $75(13 \%)$ \\
$\mathrm{C}[80]-1500$ & 459 & $343(75 \%)$ & $116(25 \%)$ \\
$\mathrm{C}[80]-2000$ & 279 & $268(96 \%)$ & $11(4 \%)$ \\
$\mathrm{C}[80]-2500$ & 195 & $172(88 \%)$ & $23(12 \%)$ \\
\hline
\end{tabular}




\section{Figure Captions}

Fig. 1: TEM images of nano-porous graphitized carbons. (a) C[80]-1000, (b) C[80]-1500, (c) $\mathrm{C}[80]-2000$, and (d) $\mathrm{C}[80]-2500$. High resolution TEM images are also displayed. (e) $\mathrm{C}[80]-2000$ and (f) $\mathrm{C}[80]-2500$.

Fig. 2: Galvanostatic lithium intercalation and de-intercalation curves of nano-porous graphitized carbon $\mathrm{C}[80]-2000$ (a) and C[80]-2500 (b). recorded at each current density.

Fig. 3: (a) Nyquist plots of impedance spectra of C[80]-2500 recorded at the 1st lithium insertion process and after the galvanostatic Li intercalation/de-intercalation test $(37.2,186$ and $372 \mathrm{~mA} \mathrm{~g}^{-1}$ for 3 cycles each, total 9 cycles). (b) is the same plot with wide range. 

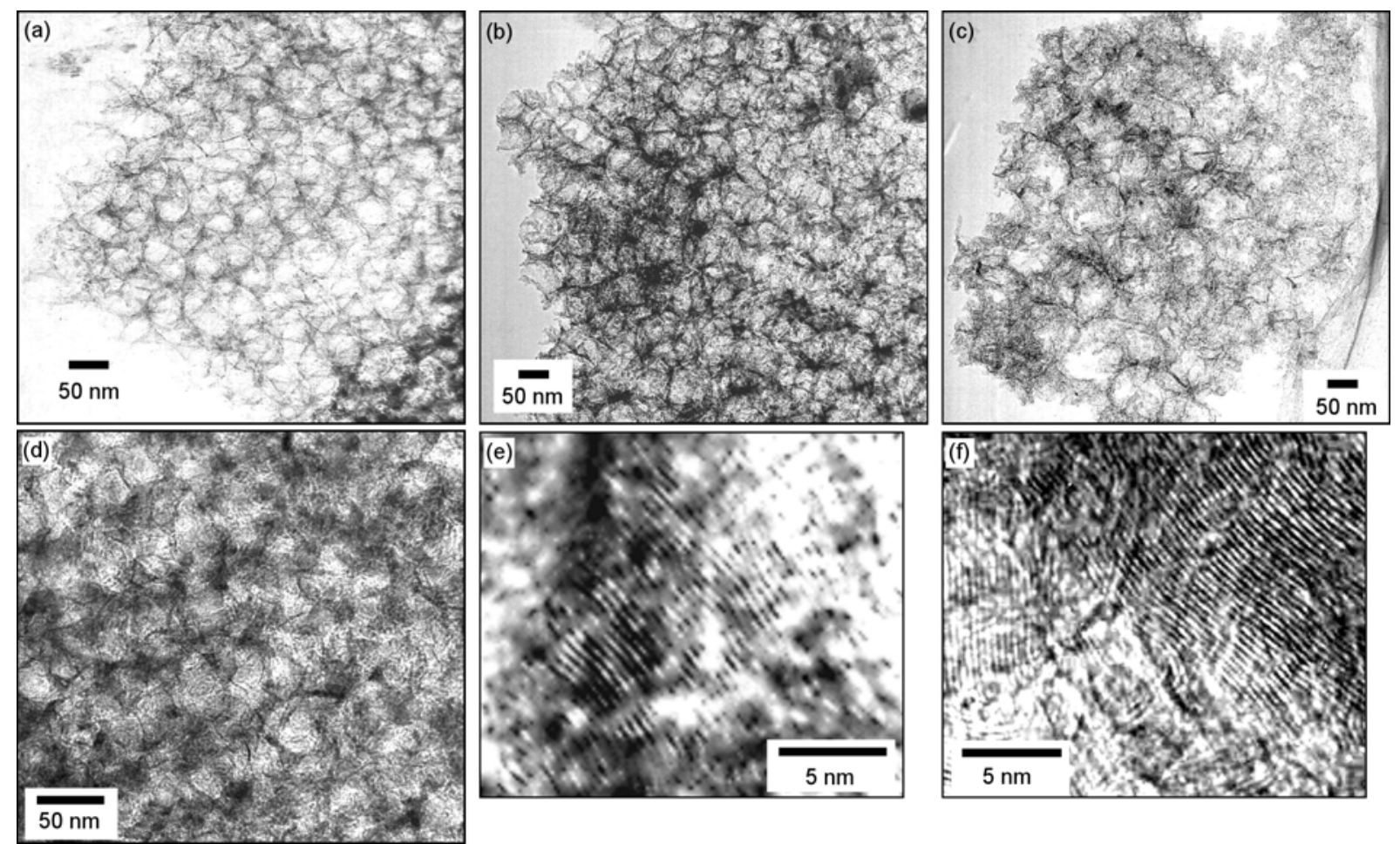

Fig. 1 


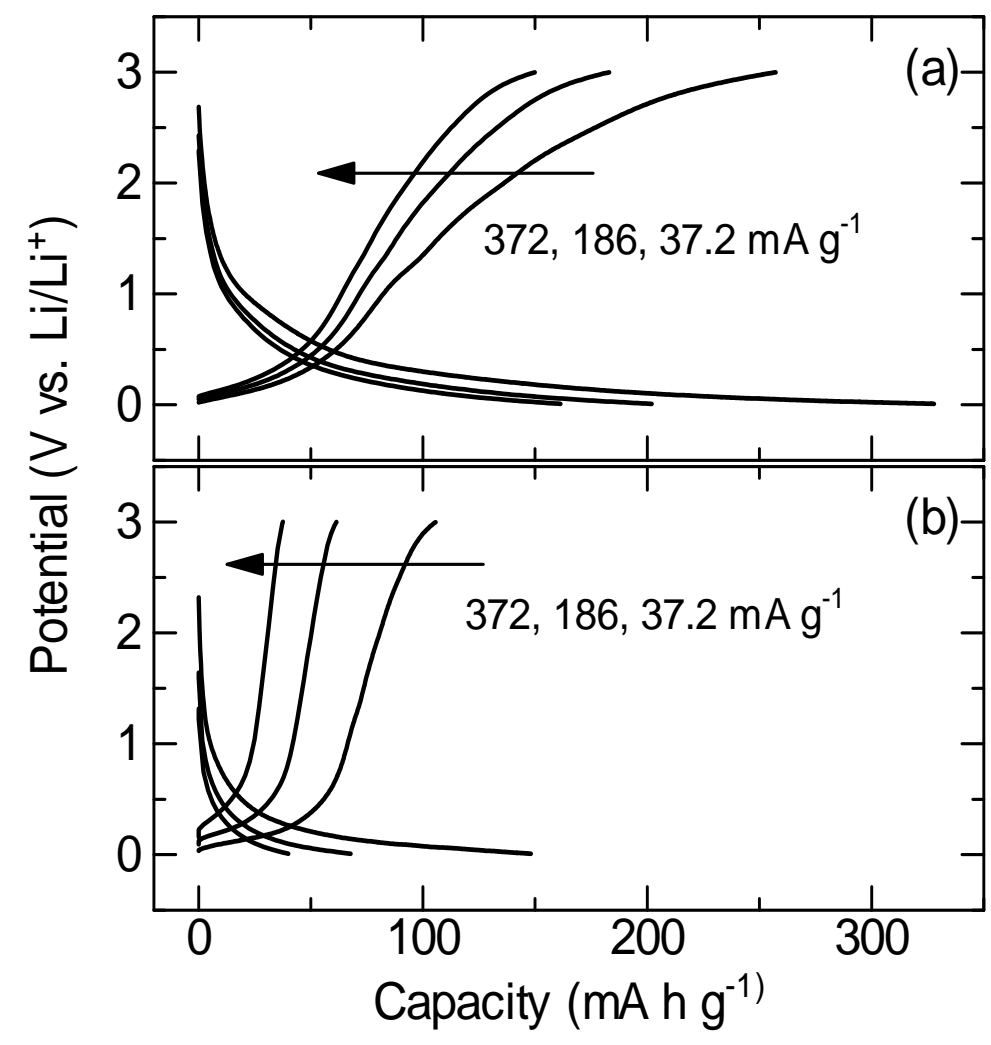

Fig. 2 

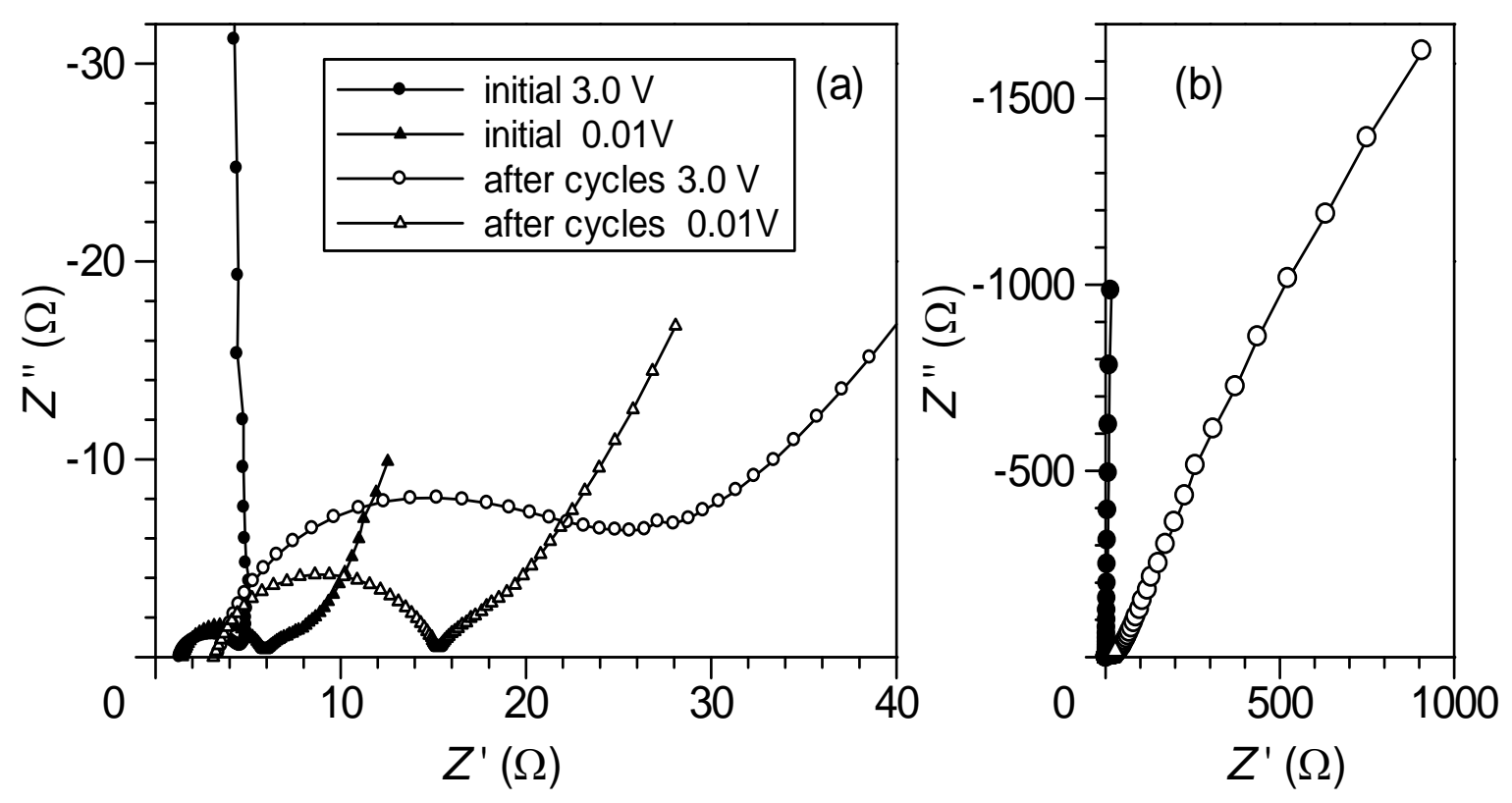

Fig. 3 